ESJ Humanities

\title{
Le chaos borgésien entre réel et virtuel
}

\author{
Majida Sayegh
}

Faculté des Lettres et des Sciences Humaines (Section 5)

Département de Langue et Littérature Françaises, Université Libanaise

Doi:10.19044/esj.2021.v17n36p98

Submitted: 02 September 2021

Accepted: 15 September 2021

Published: 31 October 2021
Copyright 2021 Author(s)

Under Creative Commons BY-NC-ND

4.0 OPEN ACCESS

Cite As:

Sayegh M. (2021). Le chaos borgésien entre réel et virtuel. European Scientific Journal, ESJ, 17 (36), 98. https://doi.org/10.19044/esj.2021.v17n36p98

\section{Résumé}

Le corpus de cette étude est composé de trois nouvelles de Jorge Luis Borges : Le jardin aux sentiers qui bifurquent, Funes ou la mémoire et Le livre de sable. L'auteur a semé des indications chaotiques à travers ces textes, une sorte de ramifications infinies dans le temps en prouvant que les événements ne sont que des points de départ pour des trajectoires bifurquées. Pour Borges, les choix humains sont des actions qui peuvent se produire selon des possibilités infinies dans un immense univers labyrinthique. Chaque décision mènerait à des parcours qui diffèrent dans le temps et qui peuvent converger, se croiser ou diverger avec d'autres qui ont débuté dans un passé ou auront lieu dans un futur lointain. A travers les mondes imaginaires de ces trois nouvelles, l'auteur veut prouver que tout n'est pas dû au hasard et nous invite à considérer la réalité comme une issue d'un labyrinthe géant sans pour autant nier la virtualité. Selon une méthode synthétique, on a pu déceler l'affinité entre le texte littéraire de Borges et la théorie du chaos.

Mots-clés : Chaos, réalité, labyrinthe, infini, bifurcation, mémoire, livre 


\title{
Borgesian Chaos Between Reality and the Virtual
}

\author{
Majida Sayegh \\ Faculty of Letters and Human Sciences (Branch 5) \\ Department of French Language and Literature, Lebanese University
}

\begin{abstract}
The essence of this study consists of three short stories by Jorge Luis Borges: The Garden of Forking Paths, Funes the Memorious, and The Book of Sand. The author has shown chaotic indications throughout these texts. A kind of infinite ramifications in time by proving that events are only starting points for branching trajectories. For Borges, human choices are actions occurring in infinite possibilities within an immense labyrinthine universe. Each decision would lead to paths that differ in time and that may converge, intersect or diverge with others that began in the distant past or will take place in the future. Through the imaginary worlds of these three short stories, the author wants to prove that not everything is due to chance, and invites us to consider reality as a way out of a giant labyrinth without denying the virtual. Using a synthetic method, the similarity between Borges' literary text and chaos theory was detected.
\end{abstract}

Keywords: Chaos, reality, labyrinth, infinite, forking, memory, book

\section{Introduction}

Selon Albert Einstein, la faculté d'imaginer est « plus importante que le savoir» ${ }^{1}$. A travers les trois nouvelles, objet de cet article, Borges réussit à mêler imaginaire et savoir. En se servant indirectement d'une notion complexe telle que la théorie du chaos, il démontre encore une fois que même les idées scientifiques les plus complexes peuvent être abordées d'une manière littéraire et même avec le fantastique, spécialité qui lui est chère.

Il semble a priori que le but de Borges n'est pas de faire plonger le lecteur gratuitement dans un monde irréel, désordonné et sans horizon, mais au contraire de lui faire ressentir la différence avec une réalité réductrice pour découvrir les lois qui gèrent le monde et qui peuvent apparaître comme chaotiques. Dans le domaine scientifique, la théorie du chaos est devenue aujourd'hui une méthode d'analyse pour comprendre les dimensions fractales, les dépendances des systèmes dynamiques aux conditions initiales. Comment Borges en tant qu'écrivain perçoit-il cette théorie difficile à assimiler pour les non spécialistes ? Pourtant, il est certain que ce grand auteur adorait la

${ }^{1}$ https://citations.ouest-france.fr/citations-albert-einstein-288.html 
mathématique et la philosophie et il « connaissait quelques paradoxes de la philosophie et de la mathématique» (Fux, 2010). Lors de son entretien avec Georges Charbonnier (1967, p. 255-345), Borges explique « Je ne crois pas être un bon mathématicien, mais j'ai lu-j'ai relu, c'était plus importantPoincaré, Russel et quelques autres mathématiciens [...] Je ne dirai pas que je suis un mathématicien ou un philosophe, mais je crois avoir trouvé dans les mathématiques et dans la philosophie des possibilités littéraires, et surtout des possibilités pour la littérature qui me passionne le plus : la littérature fantastique ». En effet, la plupart de ses nouvelles regorgent d'allusions explicites ou implicites à des concepts tels le hasard, la probabilité, le cheminement chaotique spatio-temporel, et surtout celui de l'infini mis profondément en œuvre dans la nouvelle L'Aleph. Depuis 1960, une connexion est faite entre mathématique et littérature inventive et innovante, et la création de L'Oulipo, «Ouvroir de Littérature Potentielle ${ }^{2}$ », un groupe composé d'écrivains et de mathématiciens, témoigne de cette interaction entre ces deux disciplines. A cet égard Perec (1969), un écrivain et verbicruciste français et membre de l'Oulipo, a écrit un roman intitulé "La Disparition " qui ne comporte aucun « $\mathrm{e} »$.

Avant d'aborder la notion du chaos chez Borges, il est nécessaire de souligner que l'objectif de cette recherche n'est pas d'analyser la complexité mathématique de la théorie du chaos, car tout simplement ce n'est pas notre domaine. Nos efforts seront déployés pour cerner l'intérêt borgésien au phénomène du chaos. En effet, le monde fictionnel de Borges est rempli d'espaces chaotiques qui visent à mettre en communication deux mondes distincts : la réalité et la virtualité.

Le concept du chaos qui est apparu au début du vingtième siècle préparait à une sorte de révolution scientifique comme celle de la théorie de la relativité générale d'Einstein. Les efforts des scientifiques visaient la définition du chaos et la vérité du désordre apparent des phénomènes. La recherche de cette vérité a débuté avec l'homme lui-même et elle sera recherchée tant qu'il y aura de la vie sur terre, mais « la vérité, vous le savez, c'est ce qui simplifie le monde et non ce qui crée le chaos $(172)^{3}$. Dans ce sens, le chaos désigne une absence ou une ignorance des règles qui gouvernent un phénomène et par conséquent cette absence masque sa vérité. Principalement, le chaos s'oppose à l'ordre, il n'est pas assimilé au hasard et il diverge de la philosophie par la consistance comme préconise Deleuze et Guattari (1991, p.45): «Le chaos n'est pas un état inerte ou stationnaire, ce n'est pas un mélange au hasard. Le chaos chaotise, et défait dans l'infini toute consistance. Le problème de la philosophie est d'acquérir une consistance, sans perdre

${ }^{2}$ L'Oulipo est une association fondée en 1960 par le mathématicien François Le Lionnais et l'écrivain et poète Raymond Queneau.

${ }^{3}$ Ibid. 
l'infini dans lequel la pensée plonge [...] Donner consistance sans rien perdre de l'infini...».

Y-a-t-il un lien entre le chaos et le désordre ? Autrement dit, le chaos est-il une antithèse de l'ordre ? Mais le désordre observé n'est-il pas une image trompeuse qui cache un ordre parfait? Si oui, le chaos n'est-il pas un ordre parfait géré par un attracteur? Et les manifestations désordonnées ne donnent-elles pas qu'une image embrouillée du phénomène qui est en train de se constituer ? Pourtant, le grand mathématicien Mandelbrot mentionne deux types de chaos « ordonné et désordonné» selon Peitgen et Richter (1986, p.157).

Quand on parle du chaos, il faut absolument parler du météorologue Edward Lorenz et son magique attracteur, un oscillateur de dimension fractale entre 2 et 3 selon Grassberger et Procaccia (1983). Dans son modèle dynamique sur la météo, Lorenz découvre que l'évolution à long terme de son système est fortement corrélée aux conditions initiales, c'est-à-dire , même une donnée infime peut bouleverser la prédiction due à un système dynamique, d'où la naissance de " l'effet papillon" selon lequel « le battement d'une aile de papillon à Pékin peut engendrer des remous dans l'atmosphère de New York $»^{4}$. En effet, personne n'a vu une tornade provoquée par le battement des ailes d'un papillon mais des variables liées à la météorologie terrestre la produisent si les conditions de leurs effets joints se produisent. Cette évolution chaotique découverte par Lorenz n'est jamais identique à ellemême et non périodique, mais «elle s'enroule en fait éternellement sur ellemême $»^{5}$ en sautant d'une aile à l'autre d'une manière erratique. En effet, des variations minimes dans ces conditions rendent la prédiction impossible à long terme, même pour le système purement déterministe car il est impossible de connaitre parfaitement les conditions initiales.

Pourtant, l'effet de papillon, comme les autres phénomènes chaotiques, écrit Gleick (1987, p. 41), « existait déjà dans le folklore :

Faute de clou, on perdit le fer ;

Faute de fer, on perdit le cheval ;

Faute de cheval, on perdit le cavalier ;

Faute de cavalier, on perdit la bataille;

Faute de bataille, on perdit le royaume!»

Après cette illustration préliminaire du chaos, revenons maintenant à Borges. Une question ordinaire s'impose : est-il possible de décrire la vérité de l'univers à l'aide d'un modèle unique en tenant compte des différentes

${ }^{4}$ Ibid.

${ }^{5}$ Ibid. p.49 
variations dans le cosmos? Pour Borges, la réponse est certainement non car l'univers est un labyrinthe lié à l'infini et les connaissances humaines sont impuissantes à y trouver une issue valable. Dans la première phrase de $L a$ Bibliothèque de Babel, Borges (1944, p. 79) débute la nouvelle par: «L'univers (que d'autres appellent la Bibliothèque) se compose d'un nombre indéfini, et peut-être infini, de galeries hexagonales ». Effectivement, il accorde toute la symbolique de l'infini à cet édifice en la décrivant comme « une sphère dont le centre véritable est un hexagone quelconque, et dont la circonférence est inaccessible». Puisque la bibliothèque désigne l'univers qui est un labyrinthe selon Borges, donc cette bibliothèque est un labyrinthe. L'univers cache une infinité de savoirs et le fait d'y penser provoque un malaise comme le dit Petroff (1999) : «Dans la mesure où les livres racontent l'univers et se confondent avec lui, la bibliothèque est, elle-même, l'univers des livres, c'est-à-dire, en cédant au vertige de la mise en abyme, l'univers de l'Univers».

Cette dimension infinie n'est qu'un signe que l'homme est en face d'un savoir infini, et il est condamné à utiliser toutes ses forces rationnelles et imaginaires pour acquérir ce qui est possible de se savoir. Dans ce contexte va la pensée de Pascal $(1957$, p.84) qui se demande « qu'est-ce que l'homme dans la nature ? A cette question, il répond que c'est « un néant à l'égard de l'infini, un tout à l'égard du néant, un milieu entre rien et tout, infiniment éloigné de comprendre les extrêmes». L'expression "un milieu entre rien et tout" désigne le centre d'un cercle, source de l'égalité entre les hommes dans leurs actions permanentes jusqu'à l'infini.

Borges insiste profondément sur l'exploitation intellectuelle de l'homme en conduisant son lecteur dans un monde labyrinthique, afin de l'égarer et aviver son désir de comprendre sa nouvelle et ses énigmes. Le lecteur vit ainsi un mélange de réalité et de fiction pour se terminer dans la réalité qui représente l'issue du labyrinthe, et ceci est confirmé par Nicolas (1999) qui estime que «Borges place ses nouvelles dans le domaine de la spéculation intellectuelle, et ne se rapproche de la réalité pratique que lors du bouclage final ». Ainsi, la dernière phrase de La Bibliothèque de Babel le prouve : «Ma solitude se console à cet élégant espoir (91)». Avec cette fin, Borges revient au point de départ en accordant à la bibliothèque une dimension réaliste en descendant du monde fantastique au monde rationnel. La même fin est constatée dans L'Immortel où un retour à la réalité donne vie au temps qui meurt dans l'état d'immortalité comme l'écrit Sayegh (2021) : "Borges distingue deux notions qui s'opposent : immortalité basée sur la certitude et la prévisibilité et mortalité fondée sur l'incertitude et l'imprévisibilté. La première génère une vie statique et l'autre une vie dynamique». Ceci explique les cris de joie du Troglodyte retrouvant le contact avec le temps réel car «il 
récupère son humanité perdue dans les méandres de l'uniformité de l'éternité »

Mais où est-il le chaos chez Borges ? A cet égard, Weissert (1991) affirme que Borges aurait devancé la science d'une trentaine d'années dans la formulation de la théorie du chaos, et ceci va avec le témoignage de Fux (2010) qui écrit «Borges a énoncé lui aussi qu'il y a des choses qu'on peut concevoir sans pouvoir les résoudre».

Cet article se propose d'examiner les textes de Borges suivant une méthode synthétique qui vise à saisir l'arrière-pensée mathématique et philosophique de l'auteur en rapport avec la théorie du chaos. Les nouvelles sur lesquelles sont basées les analyses sont : Le jardin aux sentiers qui bifurquent, Funes ou la mémoire et Le livre de sable. Les deux premières se trouvent dans Fictions (Borges, 1944) tandis que la troisième fait partie de son recueil intitulé Le livre de sable (Borges, 1975). Une grande attention sera accordée aux traces du chaos à travers l'univers borgésien afin d'explorer l'implicite de ces nouvelles : Est-ce la joie de voyager dans un monde fictionnel, imaginaire et virtuel ? Ou, derrière ce monde, existe-t-il un lien particulier entre le réel et le monde chaotique?

\section{Le jardin aux sentiers qui bifurquent}

Cette nouvelle ${ }^{6}$ de 16 pages est un livre de labyrinthe : Le jardin aux sentiers qui bifurquent raconte l'histoire d'un professeur chinois, Yu Tsun qui enseignait l'anglais en Angleterre et qui était en même temps un agent de l'Empire allemand pendant la Première Guerre mondiale. Sa tâche était d'enquêter puis de signaler l'endroit où se trouvent les artilleries du parc britannique. Son souci principal était de retrouver le sinologue, Stephen Albert, mais il a été découvert par le capitaine Richard Madden. Forcé à s'enfuir et à se cacher, Tsun s'est consacré à faire le point sur son passé en Chine, notamment sur son prédécesseur Ts'ui Pen, auteur d'un roman très particulier qui parle de construire un labyrinthe dans lequel tous les hommes se perdaient. Lorsque Tsun atteignit Stephen Albert, il fut grandement émerveillé par lui, en découvrant qu'il a soigneusement étudié l'œuvre de Ts'ui Pen, et il a réussi à déchiffrer l'énigme selon lequel le livre et le labyrinthe représentent le même ouvrage. C'était «Le jardin aux sentiers qui bifurquent $»$ dont le thème est le temps. Il s'agissait d'une œuvre littéraire qui cherche à décrire tous les résultats possibles d'un événement dont chacun d'eux conduit à des dénouements spécifiques et ceci se fait d'une manière continue et il nous rappelle des univers multiples selon l'interprétation de la mécanique quantique. La mission de l'espion est réussie mais les liens avec

\footnotetext{
${ }^{6}$ En 2010 avait lieu la vente aux enchères du manuscrit de la nouvelle « le jardin aux sentiers qui bifurquent».
} 
Albert se révèlent désastreux: Tsun élimine ce dernier mais fut arrêté et condamné à mort.

Après cette brève présentation de la nouvelle, il serait bénéfique de signaler l'opinion de Carolina Ferrer (2014) qui s'appuie sur le livre de Frank et Vosburg (1977) : " L'œuvre possède une structure de boîtes chinoises, composée de quatre boîtes. De l'extérieur vers l'intérieur, la première correspond à la nouvelle Le jardin aux sentiers qui bifurquent que nous lisons. La deuxième boîte est le livre d'histoire où le narrateur a trouvé la déclaration signée par $\mathrm{Yu}$ Tsun, un espion chinois qui travailla pour les Allemands pendant la Première Guerre mondiale. Dans sa lettre, troisième boîte, Tsun explique qu'il a assassiné Stephen Albert, un sinologue britannique, afin de communiquer à son supérieur qu'ils doivent attaquer la ville nommée Albert. La quatrième et dernière boîte correspond au roman Le jardin aux sentiers qui bifurquent écrit par Ts'ui Pên, l'ancêtre de Yu Tsun ».

Cette nouvelle ouvre la porte à des bifurcations temporelles, offre une nouvelle vision du monde et de l'action humaine. En quelque sorte, le lendemain n'est jamais déterministe et au même temps personne ne peut le prédire avec exactitude parce que d'une part il est lié à une décision prise à l'avance, et d'autre part, l'homme est incapable de le concevoir à cause des interventions de nombreux phénomènes erratiques provocant des perturbations sur ses réalisations possibles. C'est l'empreinte du chaos luimême. Dans ce sens va Borges lorsqu'il écrit : "Le Jardin aux sentiers qui bifurquent était le roman chaotique ; la phrase "nombreux avenirs (non tous) " me suggéra l'image de la bifurcation dans le temps, non dans l'espace [...] Dans toutes les fictions, chaque fois que diverses possibilités se présentent, l'homme en adopte une et élimine les autres ; dans la fiction du presque inextricable Ts'ui Pên, il les adopte toutes simultanément. Il crée ainsi divers avenirs, divers temps qui prolifèrent aussi et bifurquent (104)». Donc chaque décision prise à un moment donné constitue une sorte de "conditions initiales" selon la théorie du chaos, par conséquent il en résulte des avenirs différents. En suivant ce raisonnement, le présent n'est qu'un produit d'une décision prise à un particulier point de départ. En ce sens, chaque individu a des futurs multiples qui sont des bifurcations variées, de sorte que chacune d'elles est un point de départ pour d'autres bifurcations et les dénouements n'en sont qu'un reflet. Borges l'explique en écrivant : « ... tous les dénouements se produisent : chacun est le point de départ d'autres bifurcations. Parfois, les sentiers de ce labyrinthe convergent : par exemple, vous arrivez chez moi, mais, dans l'un des passés possibles, vous êtes mon ennemi ; dans un autre, mon ami (104)». C'est pourquoi les deux présences d'Albert et de Tsun se rejoignent, se confondent et se séparent au gré de bifurcations temporelles fantastiques. La figure suivante illustre cette situation : 
Schéma illustratif des bifurcations possibles

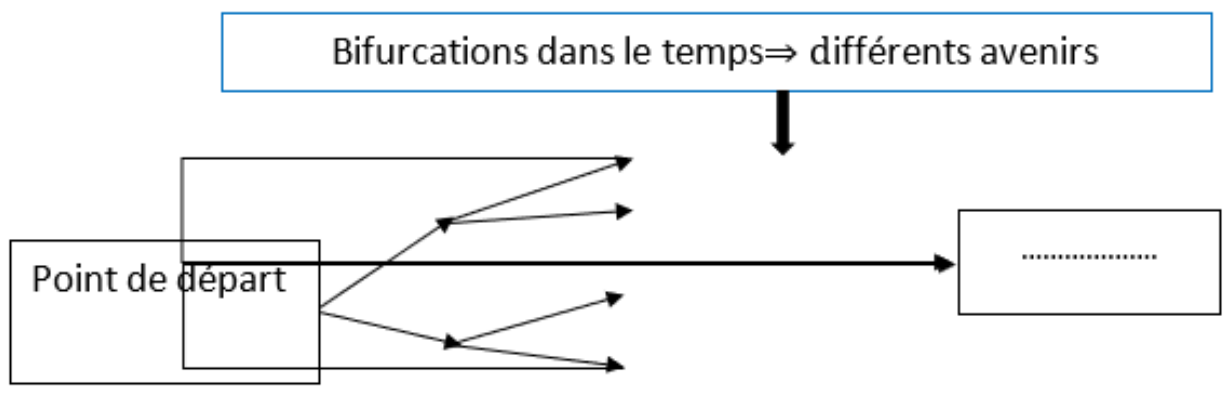

Cette analyse rejoint ce que l'écrivain Isabelle Stengers 1987)) a préconisé sur l'existence d'une multitude d'avenirs en écrivant dans l'introduction de son livre intitulé " D'une science à l'autre. Des concepts nomades " : «Le lecteur français de Borges découvre, dans Le jardin aux sentiers qui bifurquent, une parabole de la coexistence proliférante des futurs possibles, et de l'insignifiance de ce que nous croyons être et vouloir dans l'un d'entre eux [...] et il apprend la valeur métaphorique de ce mot, 'bifurcation', désignant originellement la division en deux branches d'une route».

Le narrateur a utilisé le récit de la trahison comme un prétexte pour nous conduire vers un étrange monde fictionnel. En effet l'espionnage de Tsun, personnage principal de cette nouvelle, l'a amené à examiner de multiples choix au présent pour arriver à son but qui consiste à communiquer aux allemands la bonne information concernant la base d'artillerie anglaise située en France pendant la Première Guerre mondiale. Borges nous invite à renoncer au déterminisme au profit d'une vision probabiliste selon laquelle chaque évènement dans l'univers des possibilités implique la réalisation d'un avenir différent. Cette idée est née avec la mécanique quantique découverte depuis plus d'un siècle ${ }^{7}$.

Cette multitude de choix dans le présent aboutit à une infinité de possibilités dans l'avenir : "Je pensai à un labyrinthe de labyrinthes, à un sinueux labyrinthe croissant qui embrasserait le passé et l'avenir et qui impliquerait les astres en quelque sorte (99) ». En effet, dans ce récit, la bifurcation du jardin s'ouvre à la temporalité, et conduit à une pluralité de futurs. Cette conception est confirmée par Borges quand il écrit : "Personne ne pensa que le livre et le labyrinthe étaient un seul objet (102) [...] Je m'étais demandé comment un livre pouvait être infini. Je n'avais pas conjecturé d'autre procédé que celui d'un volume cyclique, circulaire. Un volume dont la dernière page fût identique à la première, avec la possibilité de continuer indéfiniment. Je me rappelai aussi cette nuit qui se trouve au milieu des 1001 Nuits, quand la reine Schéhérazade (par une distraction magique du copiste)

7 https://lejournal.cnrs.fr/billets/peut-tester-les-univers-paralleles). 
se met à raconter textuellement l'histoire des Milles et Une Nuits, au risque d'arriver de nouveau à la nuit pendant laquelle elle la raconte, et ainsi l'infini (103)».

Autrement dit, les réalisations futures possibles ne sont pas certaines et chacune représente une bifurcation propre qui fait errer le lecteur dans un labyrinthe, par conséquent un réseau de labyrinthes parallèles se forme et l'ensemble devient un labyrinthe de labyrinthes, et pourquoi pas un ordre parce que le désordre du désordre ne signifie-t-il pas un ordre parfait ? Hugh Everett, un physicien et mathématicien américain estime que « tout ce qui aurait pu avoir lieu dans le passé a bien eu lieu dans un autre univers. Et tout ce qui peut avoir lieu dans le futur aura bien lieu dans des univers parallèles ${ }^{8}$, par conséquent des mondes multiples se créent et les prédictions changent avec chaque bifurcation. L'investigation profonde de cette nouvelle révèle que Borges avait une profonde réflexion sur les temps multiples, la multitude des avenirs possibles, des univers parallèles et des uchronies ${ }^{9}$. Borges ajoute : «Un labyrinthe minuscule... Un labyrinthe de symboles, corrigea-t-il. Un invisible labyrinthe de temps (102) ». Il ne s'agit pas d'un labyrinthe matérialisé construit par des architectes d'une grande performance dans la maximisation de l'errance, mais un labyrinthe invisible construit mentalement et son théâtre c'est le temps et non l'espace, une sorte de bifurcation des temps multiples, et l'évocation de l'œuvre orientale Les Mille et Une Nuits avait pour but de fixer cette notion dans l'esprit du lecteur. Dans chacune des nuits, Schéhérazade a mené une bataille atroce qui a duré mille et une nuits consécutives, et chaque nuit était en face de deux éventualités : la survie par une action judicieuse ou la fatalité de la mort promise par le sultan. A cet effet s'émerge implicitement des univers temporels parallèles indépendants qui forment une sorte de labyrinthe temporel, où les temps multiples s'émergent au sein de son aspect linéaire et cyclique. Pour Borges, le présent englobe tous les évènements du passé et du futur, donc la notion du temps continu a été brisée. Avec ce sens, le temps n'est plus un index des phénomènes temporels car il a l'éternité comme étendue et ses différentes formes bifurquent sans cesse.

Pour bien comprendre les sentiers mouvants de ce jardin mystérieux, il serait utile de mentionner une constellation Temps ${ }^{10}$ qui unit un grand groupe d'écrivains, de scientifiques et d'artistes dont chacun perçoit le sens du temps à sa manière. En effet, une investigation de cette constellation avec des itinéraires différents montre à quel degré la perception du temps peut dépendre

\footnotetext{
${ }^{8}$ Ibid.

${ }^{9}$ En effet selon le principe de l'uchcronie, s'il n'y avait pas la Première Guerre mondiale, l'histoire de l'humanité aurait une autre trajectoire.

${ }^{10} \mathrm{http} / / /$ www.surgissantes.com/link?id=923\&constellation_id=144
} 
de facteurs variables pour aboutir à des notions très dissemblables. Ce passage en revue offre au lecteur la possibilité de comparer plusieurs concepts du temps qui sont parfois complexes, convergents ou divergents. Ils fonctionnent de manière très variée : parallèle, linéaire, cyclique, périodique, spiral ou de diverses formes qui s'émergeront avec la progression de la pensée humaine en fournissant plus de significations pour l'existence humaine et son rôle dans cet univers. On retrouve quatre concepts dans la constellation Temps :

Pour certains le temps s'écoule et il est irrécupérable et tout ce qui se passe s'en va et ne se répète pas, telle la vision d'Apollinaire dans son poème «Le Pont Mirabeau » ou la fameuse formule d'Héraclite «On ne se baigne deux fois dans le même fleuve ».

D'autres perçoivent le temps en couleurs, baigné dans la lumière ou l'obscurité en ressentant même sa température ; il devient presque une sorte de sensation physique ou un état d'âme, mais surtout pas quelque chose de révolu et de mort. Il devient renouvelable et ressenti comme vivant ${ }^{11}$. C'est le cas du peintre impressionniste Claude Monet qui souligne le passage du temps et le changement du climat à travers ses nuances. La répétition des saisons et des couleurs sont les signes d'une renaissance toujours nouvelle malgré la ressemblance. En effet, la saisonnalité est perçue comme preuve du temps palpable qui est toujours nouveau au contraire du temps qui se répète à l'identique. Ainsi, le temps redonne vie aux choses et aux êtres au lieu d'en détruire.

Cette complicité entre le temps et la vie prend fin avec la troisième vision qui insiste sur le temps dévorant symbolisé par Cronos. Baudelaire, dans ses luttes et ses défaites continuelles contre le temps illustre l'exemple parfait de ce combat inégal contre un prédateur démesuré : pour lui, il est tout simplement l'Ennemi et résume le décalage incommensurable entre la finitude de l'homme et l'éternité du temps.

Quant aux scientifiques, leur vision du temps essaie de le priver de toute aura imaginaire ou esthétique et le perçoivent exclusivement selon leurs postulats rationnels. Il devient pour eux une entité évolutive et mouvante en interdépendance avec la notion de l'espace. Un point commun avec Borges qui a su formuler cette interférence dans ses nouvelles en faisant de l'espace la matière qui modèle l'idée abstraite du temps. Pour Albert Einstein, le temps ne s'écoule pas, il est gelé, et ce n'est que la quatrième dimension de l'espacetemps. Donc dire que le temps s'écoule c'est exhorter l'illusion : «Pour nous, physiciens dans l'âme, la distinction entre passé, présent et futur ne garde que la valeur d'une illusion, si tenace soit-elle » écrit Bouton (2018). Pour le physicien, Hawking (1989) « il fallut abandonner l'idée d'un temps unique et absolu, (180) ». Pour lui, « le désordre ou l'entropie croit toujours avec le

${ }^{11}$ Ibid. 
temps, (182) ». Ceci l'a poussé à parler de « la flèche du temps ». Pour Hegel et selon Bouton $(2015,125-141$.) le penseur de la téléologie d'une «Histoire universelle », le temps a un caractère dialectique. Pour lui, le présent appelé aussi maintenant «se nie dans l'avenir, qui se nie à son tour dans le passé, pour laisser la place à un nouveau maintenant [...] Le temps est caractérisé par deux concepts, "l'infini" et "le négatif". Le négatif désigne l'aspect destructeur du temps, sa négativité ».

Enfin, pour l'écrivain et philosophe Mikhaïl Naimy (1973, p.206), le temps ressemble au cours d'un grand fleuve et il arrive à une conclusion de nature philosophique selon laquelle la conquête du temps ne sera achevée que si l'homme est capable de ramer à contre-courant afin non seulement d'atteindre ses sources mais aussi de les dépasser pour arriver là où il n'y a ni temps ni espace.

Comme constaté dans l'étude de cette nouvelle, le temps qui règne n'est pas une ligne uniforme ou linéaire tel qu'on considère dans les études chronologiques. En effet Borges nous dit: « Cette trame de temps qui s'approchent, bifurquent, se coupent ou s'ignorent pendant des siècles, embrasse toutes les possibilités. Nous n'existons pas dans la majorité de ces temps ; dans quelques-uns vous existez et moi pas ; dans d'autres, moi et pas vous ; dans 'd'autres, tous les deux (107)».

Donc le temps borgésien qui est « un invisible labyrinthe de temps » coupe toutes les autres lignes de la constellation Temps, c'est une coordonnée de l'espace. En résumé, l'attitude de Borges par rapport au temps apparait comme l'indique Loichot (1996) : «Labyrinthe clos par sa circonférence mais infini en son sein, où se manifeste le mouvement incontrôlable du temps ... ». Et il continue que Borges «préfère la brièveté et la complétude de la nouvelle pour l'y enserrer comme dans un labyrinthe ».

\section{Funes ou la mémoire}

Dans cette nouvelle, Borges commence son récit par l'histoire d'un homme, « un précurseur des surhommes (114) » qui symbolise une capacité étrange dans le monde humain. Qu'est-ce qui distingue cet homme ? Et qu'estce qui a poussé Borges à le prendre comme modèle afin de prouver une idée du monde fantastique ? En effet, l'écrivain le présente comme unique : « ...un seul homme au monde eut ce droit (et cet homme est mort) un passionnaire sombre à la main, voyant cette fleur comme aucun être ne l'a vue, même s'il l'a regardée du crépuscule de l'aube au crépuscule du soir, toute une vie entière (113) ». C'est Ireneo Funes, un jeune indien, taciturne, mais qui possède énormément de savoirs. Il est aussi mystérieux comme le «vague paysage lacustre (113) ». Le narrateur ne l'a pas vu plus de trois jours, et il trouve son bonheur à parler de lui dans quelques pages qui seront peut-être les plus courtes, mais jamais neutres. A la question «Quelle heure est-il Ireneo 
?» Il répond directement : «Dans quatre minutes, il sera huit heures $[\ldots]$ Ainsi, il ne fréquentait personne et il savait toujours l'heure, comme une montre [...] Un chronométrique Funes (115)».

L'une des caractéristiques de Funes est que son écriture révèle une certaine ambiguité, à première vue. On dirait qu'il ne sait pas écrire et même qu'il mélange les lettres « i pour y, j pour g. [...] que cela faisait partie des bizarreries d'Ireneo (117)». Aussi dans sa chambre obscure, « Ireneo passait habituellement les heures mortes sans allumer la bougie (118) ». Funes « fut renversé par un cheval pie, [...] Pendant dix-neuf ans, il avait vécu comme dans un rêve [...] Dans sa chute il avait perdu connaissance ; quand il était revenu à lui, le présent ainsi que les souvenirs les plus anciens et les plus banals étaient devenus intolérables à force de richesse et de netteté (119) ». Sa mémoire devenue prodigieuse rappelle Cyrus, un roi perse dans l'antiquité, qui était capable d'appeler par leur nom tous les soldats de son armée et Mithridate, un roi romain de vingt-deux peuples, était capable d'haranguer chacun d'eux dans sa langue, sans interprète comme indique Borges :

" D'un coup d'œil, nous percevons trois verres sur une table; Funes, lui, percevait tous les rejets, les grappes et les fruits qui composent une treille. Il connaissait les formes des nuages austraux de l'aube $d u$ trente avril mil huit cent quatre-vingt-deux et pouvait les comparer au souvenir des marbrures d'un livre en papier espagnol qu'il n'avait regardé qu'une fois et aux lignes de l'écume soulevée par une rame sur le Rio Negro la veille du combat du Quebracho. [...]. Il pouvait reconstituer tous les rêves, tous les demi-rêves. Deux ou trois fois il avait reconstitué un jour entier; il n'avait jamais hésité, mais chaque reconstitution avait demandé un jour entier. Il me dit : J'ai à moi seul plus de souvenirs que n'en peuvent avoir eu tous les hommes depuis que le monde est monde et aussi : Mes rêves sont comme votre veille. Et aussi vers l'aube:Ma mémoire, monsieur, est comme un tas d'ordures. Une circonférence sur un tableau, un triangle rectangle, un losange, sont des formes que nous pouvons percevoir pleinement (119-120)».

Avec les formes géométriques classiques, Funes cite aussi qu'il « avait imaginé un système original de numération et qu'en très peu de jours il avait dépassé le nombre vingt-quatre mille. Il ne l'avait pas écrit, car ce qu'il avait pensé une seule fois ne pouvait plus s'effacer de sa mémoire (121)».

Il est clair que Borges raconte un récit qualifié de « doublement chimérique » car c'est une fiction et un personnage de fiction comme souligne Otero (2006). Il s'agit d'un jeune indien possédant une mémoire d'une capacité inimaginable qui dépasse les mémoires de tous les hommes réunis. Dans son œuvre Fictions, Borges le décrit comme suivant : «En effet, non 
seulement Funes se rappelait chaque feuille de chaque arbre de chaque bois, mais chacune des fois qu'il l'avait vue ou imaginée (122)». Funes invente son propre système de numération, une liste apparemment chaotique et non arbitraire, par exemple (121) :

$$
\begin{aligned}
7013 & \Leftrightarrow \text { Maxime Pérez } \\
7014 \Leftrightarrow \text { Le chemin de fer } & \\
& 500 \Leftrightarrow 9
\end{aligned}
$$

«Ireneo avait dix-neuf ans ; il était né en 1868 [...] mourut en 1889 (124) » : un simple calcul informe qu'il avait vingt-et-un ans. S'agit-t-il d'une erreur arithmétique ? Nous ne le croyons pas. La seule interprétation possible est que le nombre dix-neuf chez Funes correspond à son propre système de numération qui n'est pas le même chez le narrateur.

Cet indien possède une mémoire exhaustive privée de toute perturbation possible ce qui lui permet de citer son vécu d'hier sans erreur avec les détails les plus minimes, seconde par seconde. Cette étrange révélation par Borges indique que Funes détient une mémoire infaillible avec une vision complète et précise des évènements qui surgissent avec un ordre parfait. En effet, les humains sont tous condamnés à l'oubli à cause des divers facteurs inter-corrélés qui réduisent la capacité de leur mémoire. Puisqu'on est exposé à une sorte de déviation de la concentration, par conséquent il est impossible d'accueillir des informations parfaites qui sont soumises à un « masque restrictif» figurant d'une manière permanente dans l'environnement intellectuel et matériel de l'homme. Ce masque est dû aux aléas des interactions humaines et des phénomènes cosmiques illimités qui influent sur notre planète, ce qui fournit aux hommes des informations confuses qui nécessitent un éclaircissement rapide sinon leur usage systématique les conduira ailleurs. Le système de numération inventé par Funes a-t-il un ordre ? Apparemment non, mais Borges n'a pas choisi ses équivalences au hasard. En effet, la capacité mémorielle illimitée de ce personnage nous laisse penser au contraire. Pour lui, il existe un ordre parfait dans ces nombres choisis et seule sa mémoire est capable de le déchiffrer.

Une question fondamentale se pose : où est le chaos dans le système de numération de Funes? Est-ce dans sa mémoire ou dans sa vision explicite du monde ? Comme mentionné ci-dessus, Funes a une mémoire égale ou supérieure à celle de tous les hommes réunis depuis l'existence du monde humain. Tout simplement, cela ne s'explique que du point de vue de la théorie du chaos elle-même : nous ne pouvons pas diagnostiquer l'attracteur chaotique dont dispose Funes car il voit exactement ce qu'il a du «chaos ordonné » comme l'appelle le mathématicien Mandelbrot. Cela est clairement démontré dans sa personnalité et son potentiel mental qui ont été décrits dans cette nouvelle. Cela signifie que Funes dispose d'un attracteur spécifique à lui, qui 
le rend capable d'amener des désordres apparents à un ordre parfait. C'est l'ordre dans le désordre, et dans un langage scientifique moderne inspiré de la théorie du chaos, sa mémoire voit l'attracteur caché derrière ces nombres.

\section{Le livre de sable}

Le titre est souvent évocateur chez Borges. Le livre de sable raconte l'histoire d'un «livre infini (131)»: le narrateur achète d'un vendeur de Bibles un livre fabuleux contenant tous les livres. A l'instar de la bibliothèque de Babel $^{12}$, ce «Livre des livres (129» est incapable de fournir une quelconque information cohérente car «le nombre de pages de ce livre est exactement infini. Aucune n'est la première, aucune n'est la dernière ». Terrorisé par le pouvoir chaotique de ce livre, le narrateur décide de s'en débarrasser.

Le cadre de la nouvelle semble réel. Tout a commencé avec l'arrivée de ce vendeur étranger originaire des Orcades chez le narrateur habitant au quatrième étage d'un immeuble de la rue Belgrano. Au premier abord, le colporteur avec son profil et sa valise grise semble surgir de nulle part tout comme le livre sacré qu'il essaie de vendre et qui s'appelait « le livre de sable parce que ni ce livre ni le sable n'ont de commencement ni de fin (130)». Comment comprendre cette déclaration ? En effet, entrer dans ce livre, c'est comme entrer dans un labyrinthe de sable dans «une déambulation labyrinthique, sans véritable début ni fin. Le sable, balayé par les pas des visiteurs, dessine et redessine indéfiniment le paysage proposé ${ }^{13} \gg$. L'analogie entre le livre et le sable incarne l'infini et donc un labyrinthe. En effet, le contenu du Livre de sable est indéfini et insaisissable par l'esprit humain. Les informations engendrent d'autres et avec une ramification illimitée causant ainsi le vertige.

Dans cette nouvelle, Borges entre dans l'espace géométrique en citant des objets dont chacun contient une infinité d'autres objets. Ceci crée une profonde angoisse chez le lecteur en le plaçant devant la porte de l'infini, en commençant par la droite ayant une seule dimension et en terminant par l'hyper volume de dimension supérieure à trois : « La ligne est composée d'un nombre infini de points, le plan, d'un nombre infini de lignes, le volume, d'un nombre infini de plans, l'hyper-volume, d'un nombre infini de volumes (127)». C'est un début étonnant qui évoque le thème de l'infini cher à Borges qui tient le livre non seulement entre ses mains, mais par son esprit assoiffé de mystères. Par ces indications, il a l'intention de nous informer sur cette nouvelle labyrinthique avant d'y entrer. En investiguant les connotations de ces éléments géométriques et l'ordre de leur évocation, il est constaté que

\footnotetext{
${ }^{12}$ La Bibliothèque de Babel p.79 (Fictions)

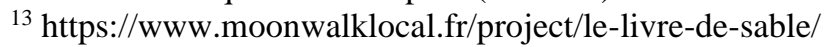


Borges exploite le passage entre ces objets géométriques du fait que le passage de la ligne au plan fait augmenter la dimension d'une unité, de même le passage du plan au volume et du volume à l'hyper-volume. L'univers plan contient l'univers d'une droite, et l'univers volume contient l'univers des plans, et enfin l'univers de l'hyper volume contient des volumes. En effet, le passage d'un objet géométrique à l'hyper-volume, ${ }^{14}$ qui est un objet supérieur, fait accroître la complexité et la possibilité de mouvement. En feuilletant ce livre infini, la seule information donnée par Borges est que «sur l'une des pages, [il vit] le dessin d'un masque. Le haut du feuillet portait un chiffre, qu'[il a oublié], élevé à la puissance 9 (132)».

Dans l'épilogue du Livre de sable, Borges nous informe qu'il s'agit d'un « volume au nombre incalculable de pages (137)». Autrement dit, il contient une infinité de pages et non une quantité physique de pages imprimées avec des caractères à l'encre. Ceci prouve le raisonnement selon lequel la quantité d'informations surgie de ce livre se ramifie indéfiniment pour remplir un champ infini du savoir. Puis Borges révèle une étrange description de son livre: «À l'angle supérieur des pages figuraient des chiffres arabes. Mon attention fut attirée sur le fait qu'une page paire portait, disons, le numéro 40514 et la page impaire qui suivait, le numéro 999. Je tournai cette page; au verso la pagination comportait huit chiffres (129)». Puisque les numéros de pages se partagent entre les paires et les impaires, les numéros 40514 et 999 ont chacune une chance de $50 \%$ d'être choisi au hasard, une distribution uniforme de probabilité et par suite l'incertitude est maximale d'après la théorie de l'information de Shannon (1948). Cette pagination mystérieuse semble due au hasard, et la lecture d'une page accable complétement le lecteur en réduisant au strict minimum sa capacité de comprendre car cette page est certainement liée aux informations écrites dans d'autres pages qui la précèdent véritablement et non selon cette numération arbitraire choisie pour ce livre. Cela signifie que les pages sont indifféremment interchangeables dans ce volume chaotique où le début et la fin n'existent pas : « Le nombre de pages de ce livre est exactement infini. Aucune n'est la première, aucune n'est la dernière (130) ». Cela implique que la chance d'une page d'être la première ou la dernière, est nulle, de plus, la chance de trouver une page à sa vraie place est aussi nulle. De là, rechercher une page spécifique de ce livre serait comme chercher un grain de sable dans un monde qui contient un nombre infini de grains de sable. Nous sommes dans deux labyrinthes parallèles : le premier résulte des pages paires qui sont représentées par le numéro 40514 et le second est dû aux pages impaires portant le numéro 999. En effet, même si quelqu'un dispose d'une information parfaite selon laquelle un thème particulier est écrit sur une page paire par exemple, chercher dans cette catégorie de pages, c'est

\footnotetext{
14 Toute existence supérieure à la troisième dimension
} 
comme chercher une issue dans un labyrinthe infini. Cette situation accroit la confusion du lecteur et génère en lui le plus haut degré de dégoût. Cela signifie que chaque page peut être dans n'importe quelle place, comme le confirme Borges en disant: "Les composants d'une série infinie peuvent être numérotés dans n'importe quel ordre [...] Si l'espace est infini, nous sommes dans n'importe quel point de l'espace. Si le temps est infini, nous sommes dans n'importe quel point du temps (130) ». C'est un livre monstrueux et absurde car le narrateur constate que « les petites illustrations se trouvaient à deux mille pages les unes des autres (132-133) ». C'est une déclaration nette d'une répétition illustrative de période de 2000 pages. Cet ordre n'a qu'une seule interprétation selon laquelle un point de départ se crée chaque 2000 page où une bifurcation surgit et par conséquent l'univers de ce livre est chaotique, or la force intellectuelle de l'homme est incapable de saisir l'attracteur de ce livre chaotique. Après l'avoir examiné en profondeur, le narrateur le qualifie d'un « objet de cauchemar, une chose obscène qui diffamait et corrompait la réalité (133) » et décide de le brûler mais il craignait que « la combustion d'un livre infini ne soit pareillement infinie et n'asphyxie la planète par sa fumée (133) », c'est pourquoi il s'en débarrasse en le classant « sur l'un des rayons humides (133) » de la Bibliothèque nationale.

\section{Conclusion}

A travers ses voyages dans les mondes imaginaires représentés par les chemins fourchus de son jardin virtuel, l'immense capacité de la mémoire du jeune indien Funes et dans sa vision cauchemardesque et labyrinthique du Livre de sable, Borges a pu concevoir l'existence du chaos avant que les spécialistes le découvrent et le formulent à l'aide de leurs sciences théoriques et expérimentales. Il veut prouver que les choix initiaux des humains aboutiront inévitablement à des chemins infiniment bifurqués de sorte que l'imagination et le réel se confondent, par conséquent la réalité et la virtualité deviennent une seule pièce à double face. Il va de soi que Borges est entré dans le domaine de la fiction non pas pour faire perdre le lecteur dans son monde infini, mais pour annoncer la présence d'une issue de sauvetage à travers la réalité elle-même. En effet, à la fin de chacune de ses nouvelles, Borges intervient directement pour sauver le réel des griffes du virtuel et nous faire revenir dans le monde humain limité par toutes les finitudes malgré le désir suprême de déceler les secrets de l'existence labyrinthique.

L'imagination de Borges ne se limite pas à l'espace et au temps, et sa culture littéraire ouverte aux mathématiques, à la philosophie et à l'histoire humaine en tout temps et en tout lieu, lui a permis de ressentir la présence de ces différents univers auxquels les spécialistes des sciences du hasard, de la probabilité et du chaos accordent une importance capitale. Borges a abordé ces univers en utilisant une forme littéraire basée sur la fiction et les symboles 
et dépourvue de toute véracité scientifique ce qui fait de lui un précurseur dans son domaine : il a innové et enrichi la littérature en l'ouvrant profondément aux théories scientifiques fondamentales touchant la vision humaine de l'existence.

Tous les points de vue rapportés, dès le scientifique Albert Einstein sur l'imagination qui dépasse le savoir dans son importance, au Stephen Hawking et son concept de la flèche du temps, au Hegel et sa vision dialectique du temps, aux constellations d'écrivains et de peintres qui ont exprimé leurs visions du temps, mettent l'accent sur les ramifications et la diversité des concepts du temps. Tous ces fondements intellectuels sont, d'une manière ou d'une autre, des témoignages sur les bifurcations annoncées par la pluralité borgésienne du temps.

Enfin, le recours à la théorie du chaos, dans ses grandes lignes, dont la compréhension constitue une grande difficulté pour un non spécialiste n'était qu'un adjuvant pour essayer de saisir la pensée borgésienne dans ce domaine. Indirectement, Borges ouvre grande la porte de la littérature à la virtualité et aux théories scientifiques afin d'aider l'homme à sortir des sentiers battus qui limitent les visions humaines. Ainsi le recours au virtuel semble un moyen d'enrichir le réel au lieu de le remplacer.

\section{References:}

1. Borges, J. (1944). Fictions. Paris: Folio: (Édition Française, 1993).

2. Borges, J. L. (1975). Le livre de sable. Traduction revue par JeanPierre Bernes. Paris: Gallimard,1978.

3. Bouton, C. (2015). La dialectique du temps. Publié dans le livre: Hegel à Iéna. ENS Éditions.

4. Bouton, C. (2018). Le futur est-il déjà présent ? La théorie de la relativité restreinte et la conception de l'« univers bloc ». Etudes philosophiques sur le temps dans les sciences naturelles, Paris, CNRS, 115-148.

5. Charbonnier, G. (1967). Entretiens avec Jorge Luis Borges, in Jorge Luis Borges, Enquêtes. Paris: Gallimard.

6. Deleuze, G., \& Guattari, F. (1991). Qu'est-ce que la philosophie? (éd. Éditions de Minuit). Paris.

7. Ferrer, C. (2014). De la citation apocryphe à la théorie cachée : "Le jardin aux sentiers qui bifurquent" de Jorge Luis Borges. Narrative Matters: Narrative Knowing/Récit et Savoir, Jun 2014, Paris,France. Hal-01099265.

8. Frank, R. M., \& Vosburg, N. (1977), « Textos y contra-textos en 'El jardín de senderos que se bifurcan'», Revista Iberoamericana, 43, 517533. 
9. Fux, J. (2010). La mathématique chez Georges Perec et chez Jorge Luis Borges: une étude comparative. Campinas-SP, 30(2), 259-276.

10. Gleick, j. (1987, p. 41). La théorie du chaos. Vers une nouvelle science. Paris: Editions Albini Michel S.A., 1989 pour la traduction française.

11. Grassberger, P., \& Procaccia, I. (1983). Measuring the strangeness of strange attractor. Physica D: Nonlinear Phenomena, 9(1-2), 189-208.

12. Hawking, S. (1989, p.180). Une breve histoire du temps: Du big Bang aux trous noirs. Paris: Flammarion.

13. Loichot, V. I. (1996). Le Temps Dans Les Oeuvres De Jorge Luis Borges, Edouard Glissant Et Saint-John Perse. LSU Historical Dissertations and Theses. 6201.

doi:https://digitalcommons.lsu.edu/gradschool_disstheses/6201

14. Naimy, M. (1973). Le Dernier jour (version en arabe). Collection complète.Tome 2. Beyrouth: Dar El Ilm Lilmalayin.

15. Nicolas, L. (1999). Borges et l'infini. Variaciones Borges(7), 88-146.

16. Otero, L. (2006). Jorge Luis Borges et Paul Valéry : Ireneo Funes et Edmond Teste ou deux visions monstrueuses de l'idéal. Revue de littérature comparée, 4(320), 475-490. Récupéré sur https://www.cairn.info/revue-de-litterature-comparee-2006-4-page475.htm

17. Peitgen, H.-O., \& Richter, P. H. (1986, p.157). The Beauty of Fractals.Images of Complex Dynamical Systems. Berlin: Springen.

18. Perec, G. (1969). La Disparition. Paris: les Lettres nouvelles.

19. Petroff, M. (1999). Jorge Luis Borges (1899-1986) la bibliothèque $e(s) t$ le labyrinthe. Suisse: Bibliothèque publique et universitaire.

20. Sayegh, M. (2021). L'Immortel de Borges ou le refus de l'éternité . European Scientific Journal, ESJ, (17)6,130-149. https://doi.org/10.19044/esj.2021.v17n6p130

21. Shannon, C. E. (1948). A Mathematical Theory of Communication. The Bell System Technical Journal, 27, 379-423, 623-656, July, October.

22. Stengers, I. (1987). D'une science à l'autre. Des concepts nomades. Paris, Seuil

23. Weissert, T. P. (1991). Representation and Bifurcation: Borges's Garden of Chaos Dynamics. In Chaos and Order. Complex Dynamics in Literature and Science. Ed. N.Katherine Hayles. Chicago, The University of Chicago Press, 223-243.

24. https://citations.ouest-france.fr/citations-albert-einstein-288.html 25. https://lejournal.cnrs.fr/billets/peut-tester-les-univers-paralleles). 26. http://www.surgissantes.com/link?id=923\&constellation_id=144 27. https://www.moonwalklocal.fr/project/le-livre-de-sable/ 